\title{
Clinical Study of 200 Cases of Absorbable Hemostatic Gauze in Breast Cancer Surgery
}

\author{
Yanming $\mathrm{Li}^{1, \mathrm{a}}$,Yian $\mathrm{Lu}^{2, \mathrm{~b}}$ and Aizhong Zhao ${ }^{3, \mathrm{c}, \Delta}$ \\ ${ }^{1}$ Nursing college, beihua university, jilin city, jilin province, china \\ ${ }^{2}$ The first clinical medical college, beihua university, jilin city, jilin province, china \\ ${ }^{3}$ University hospital of beihua university, jilin city, jilin province, china \\ ad43789777@qq.com, b492964574@qq.com, ${ }^{c} 1185362108 @ q q . c o m$
}

\begin{abstract}
Keywords: breast carcinoma, modified radical operation, absorbable hemostatic materia.
Abstract. We intended to observe the effect of absorbable hemostatic gauze (Tai Ling) in breast cancer surgery. We selected 200 cases of modified radical mastectomy for breast cancer patients, which were randomly divided into observation group and control group with 100 cases in each group. In the observation group, the application of absorbable hemostatic gauze to stop bleeding, the control group using traditional mechanical to stop bleeding, the difference between the two groups of patients were observed. From these data, we can observe The operation time, blood loss, the number of red blood cell count, extubation time and total drainage volume of the observation group after the operation of 1day, 2days, 3days were lower than the control group, the difference was statistically significant $(P<0.05)$. From this experiment, we can sum up: Absorbable hemostatic gauze (Tai Ling) in breast cancer surgery and postoperation has a good hemostatic effect. Absorbable hemostatic can prevent the occurrence of lymphatic leakage, reducing the postoperative wound bleeding, subcutaneous effusion complications.
\end{abstract}

\section{Introduction}

Breast cancer is one of the most common malignant tumors in women. The incidence of breast cancer is increasing year by year in China. It has already risen to first place in female malignant tumor incidence, which seriously harms women's physical and mental health[1]. The main treatment method of breast cancer is to improve the radical operation of comprehensive treatment, because the surgical wounds were huge and it also carries out axillary lymph node dissection. Moreover, it is also easy to appear the complications such as wound bleeding, subcutaneous effusion and so on[2]. These complications not only affect wound healing, prolonging the hospital stay, but also increase the economic and psychological pressure, thereby affecting the operation, chemotherapy, radiotherapy, and a series of treatment, These complications affect the prognosis of patients and the effect of the comprehensive treatment in minority. Therefore, the effective control of the complications of breast cancer patients with positive significance. Absorbable hemostatic gauze is a kind of biological medical material, which can be used to stop bleeding quickly and can be absorbed by human body in a certain period of time[3]. The biggest characteristic is that it can be used in the human body or in the animal body to stop bleeding, in the same time, it can be used to stop bleeding, which can be degraded without taking out. It avoids the second damage after the completion of the removal of the wound dressing[4]. As the third kind of medical device products, the use of the requirement of absorbable hemostatic material is higher than the traditional external dressings and the risk is higher, which makes the evaluation of the products more demanding and more standard. the application of absorbable hemostatic gauze for hemostasis (Tai Ling) in the affiliated Hospital of our school about200 cases of breast cancer patients treated, which achieved good results, report as follows now. 


\section{Materials and Methods}

General Information. From May 2014 to August 2015 in our affiliated hospital for breast surgery after modified radical mastectomy of breast cancer in 200 patients as the research object, randomly divided into observation group and control group, 100 cases in each group. Observation group: age 42 to 61 years old, the average age $(52.3 \pm 2.5)$ years old; control group: 47 to 66 years old, the average age $(54.35 \pm 2.2)$ years old. The patients were female, whose breast cancer modified radical mastectomy were performed under general anesthesia, all the patients were placed a drainage tube after the operation. The patients had no diabetes, severe cardiovascular system and respiratory system disease, liver and kidney function were normal. There was no significant difference in age, body mass index, culture level, marital status, time of Symptom onset, clinical manifestation and other data of the two groups $(P>0.05)$. They had comparability.

Method. Observation group: In the surgery, the breast specimens were taken out, the axillary lymph node dissection was performed and the wound was strictly hemostasised, putting the 2 pieces of tai ling $(8 \mathrm{~cm} \times 5 \mathrm{~cm})$ into $20 \mathrm{ml}$ normal saline, completely melted evenly in the wound. control group: Intraoperative wound suturing and electrocoagulation hemostasis without using absorbable hemostatic gauze and directly suturing. All patients need to record all the indexes, collect and count the actual amount of bleeding.

Observation Index. Because the observation group was to pull the tube within 4days. We recorded the drainage flow within 1day, 2days, 3days after the operation, the red blood cell count in the drainage fluid was detected, and the time of extubation and the total amount of drainage were recorded.

Statistical Analysis. Use SPSS 15 statistical software package to establish a database, the measurement data is represented by mean and standard deviation $(\mathbf{x} \pm \mathbf{s})$.we used $\mathrm{T}$ test and the difference was statistically significant with $P<0.05$.

\section{Results}

Comparison of the Flow Amount of the Two Groups. As shown in table 1, the drainage in the observation group after the operation within 1day, 2days, 3days were less than those in the control group, the difference between the two groups was statistically significant $(P<0.05)$.

Comparison of Red Blood Cells of the Drainage in Two Groups. As shown in table 2, the number of red blood cells in the observation group was less than that of the control group after the operation within 1day, 2days, 3days, the difference between the two groups was statistically significant $(P<$ $0.05)$.

Comparison of Total Drainage and Extubation Time in Two Groups. As shown in table 3, in observation group, the total flow volume and the time of extubation were less than the control group.The difference between the two groups was statistically significant $(P<0.05)$.

Table 1, Comparison of two groups of drainage ( $\mathrm{ml}, \overline{\mathbf{x}} \pm \mathrm{s})$

\begin{tabular}{lcccc}
\hline group & $\mathrm{n}$ & ato $1 \mathrm{~d}$ & ato $2 \mathrm{~d}$ & ato $3 \mathrm{~d}$ \\
\hline obs group & 100 & $32.5 \pm 12.2$ & $80.5 \pm 5.1$ & $72.3 \pm 2.4$ \\
con group & 100 & $53.6 \pm 1.7$ & $113.4 \pm 4.2$ & $89.5 \pm 3.6$ \\
$\mathrm{t}$ & & 11.235 & 12.163 & 9.35 \\
$P$ & & $P<0.05$ & $P<0.05$ & $P<0.05$ \\
\hline
\end{tabular}

obs $=$ observation con $=$ control ato $=$ after the operation 
Table 2, Comparison of red blood cells in two groups of

Postoperative drainage fluid ( $\mathrm{ml}, \overline{\mathbf{x}} \pm \mathrm{s})$

\begin{tabular}{lcccc}
\hline group & $\mathrm{n}$ & ato $1 \mathrm{~d}$ & ato $2 \mathrm{~d}$ & ato $3 \mathrm{~d}$ \\
& & & & \\
\hline obs group & 100 & $1.17 \pm 0.32$ & $0.53 \pm 0.16$ & $0.23 \pm 0.12$ \\
con group & 100 & $2.35 \pm 0.74$ & $1.42 \pm 0.52$ & $0.91 \pm 0.31$ \\
$\mathrm{t}$ & & 11.235 & 12.163 & 9.35 \\
$P$ & & $P<0.05$ & $P<0.05$ & $P<0.05$ \\
\hline
\end{tabular}

obs $=$ observation con $=$ control ato $=$ after the operation

Table 3, Comparison of total drainage and extubation

time in two groups $(\overline{\mathbf{x}} \pm \mathrm{s})$

\begin{tabular}{lccc}
\hline group & $\mathrm{n}$ & total drainage $(\mathrm{ml})$ & extubation time $(\mathrm{d})$ \\
& & & \\
\hline obs group & 100 & $269.35 \pm 86.32$ & $5.13 \pm 2.16$ \\
con group & 100 & $330.35 \pm 96.74$ & $8.42 \pm 2.53$ \\
t & & 10.152 & 9.363 \\
$P$ & & $P<0.05$ & $P<0.05$ \\
\hline
\end{tabular}

obs $=$ observation con $=$ control

\section{Discussion}

Breast cancer surgery due to trauma, postoperative complications, such as subcutaneous effusion or bleeding complications [5]. At present, the clinical treatment of these complications is not much, and the effect is not good. However, the axillary region and the chest wall area are different, the density of the axillary lymphatic area could produce lymph exudation, whose color of the drainage liquid was pale yellow or light yellow. While the chest muscle is the main material of the chest wall, effusion was often the product of blood and tissue infiltration, mostly dark red, this area was generally not a lot of lymphatic leakage[6]. Subcutaneous effusion and hemorrhage due to many aspects: the operation of large lymphatic vessels without ligation, bleeding, etc., increasing the amount of subcutaneous fluid. The free and wide flap, and the wound lead to iatrogenic huge lacuna, It would have more tissue fluid, blood and lymph accumulation after the operation. The excessive use of intraoperative electric knife making free flap and the use of electric knife would cause uneven thickness of adipose tissue, leading to the formation of fat liquefaction of subcutaneous effusion [7]. Poor drainage tube drainage after surgery can not make lacuna narrows as soon as possible, which would aggravate the formation of effusion. Although through ligation, hemostasis, drainage, pressure dressing and other measures, there were still many patients with subcutaneous effusion, bleeding, and even the need to re set the tube, debridement suture, which not only affected the recovery of the disease, the patient's physical and mental but also had a negative impact, which is more conducive to prognosis and follow-up treatment. Therefore, effective and safe to reduce these complications, which had an important significance for breast cancer patients [8].

With the continuous development of science and technology, the biological hemostatic materials are constantly emerging, and the performance is better and better. Following the first generation (gauze for hemostasis) and the second generation (fibrin glue) hemostatic materials, the third generation of absorbable hemostatic material tailing, because of its unique advantages and widely used in clinical. The main component of tailing for regenerated cellulose extracted from natural plants, which can be quickly absorbed, hemostasised, soft, light and thin texture and during surgery it does not affect the 
operation field and operation, the recovery after operation in the process, no tissue reaction,the tailing can effectively prevent tissue adhesion, promoting the healing of the tissue [9]. Compared to the first generation and the second generation of hemostatic materials, Tai Ling without tissue rejection, no adverse reactions to the nerves and blood vessels, the final decomposition products into water and carbon dioxide, whose compositive characteristics of similar products and avoiding the shortage of animal protein products of tissue reactions (such as allergies, fever, edema, infection and other reaction) on the human body. It is more safe and reliable [10]. The bleeding of the patients with blood coagulation dysfunction and heparin were significantly. Its decomposition products can be absorbed by the human body to provide nutrients for the cell to create a low acid and low oxygen environment for the injured area. It also can promote necrosis tissue absorption and capillary, capillary lymphatic capillaries of the new [11]. The results of this study show that Tai Ling can reduce the amount of bleeding, shorten operation time, reduce postoperative drainage, wound bleeding and subcutaneous effusion, which suggests that tailing has reliable hemostasis and prevention of subcutaneous hydrops and lymph leakage[12]. The total drainage volume of the observation group was less than the control group, and the extubation time is shorter than the control group, so the Tai Ling may contribute to early wound adhesion, promoting the wound to be repaired. The results still need to be further verified by a larger and more systematic test[13]. At the same time on the tailing using method is to be improved. 2 pieces of tailing fully dissolved in saline, then spreading evenly in the wound. It can fully reduce the huge lacuna caused by breast cancer surgery, and can reduce the amount of tailing, reducing the economic burden of patients. Tai Ling can effectively prevent bleeding, subcutaneous effusion, lymph leakage, but it can not be a substitution for surgical ligation [14], so the premise of application of tailing is the operation which must be thoroughly ligated of blood vessels and lymphatic vessels, supplemented by Tai Ling, to achieve the ideal effect.

Absorbable hemostatic gauze (tailing) used in modified radical mastectomy in breast cancer, can effectively shorten the operation time, reducing the amount of bleeding, less postoperative drainage volume and shorten the extubation time, which also help to promote the wound healing, so it is worth being promoted in clinical treatment.

\section{References}

[1] Zhijun Ma: The clinical effect of breast conserving surgery and modified radical mastectomy for early breast cancer. Tumor Clinical and Rehabilitation of China Vol.11(2015), p. 320.

[2] Pangzhou Chen, Yugen Xiao and Yihong Tan: Breast conserving surgery and modified radical surgery contrast effect in the surgical treatment of breast cancer. Jilin Medical Journal Vol.5(201 4), p. 113.

[3] Huachun Liu: Application of laminectomy tailing prevention of epidural scar adhesion. Chinese Contemporary Medicine Vol.18 (2011), p. 187-190.

[4] Shaojun Ling: Tai Ling cholecystectomy diagnosis prevention of complications. Medical InformAtion Vol.3(2011), p. 924-925.

[5] Yunjun Song, Linhe Jiang and Yanli Leng: Clinical observation of 40 cases of breast conserving surgery for early breast cancer. Hebei Medical Vol.8 (2014),p. 359.

[6] Lei Feng and Henggang Zhang: Surgical treatment of skin flap necrosis after improved surgical treatment of breast cancer. Modern Oncology Vol.3(2013), p. 325.

[7 ] Shifu Ye, Shibin Wang and Wen Wu: Research progress of absorbable hemostatic materials to evaluate the status and effect. Materials Review Vol.28 (2014), p. 68.

[8] Zhongxiao Zhou, Xilin Wei and Jianru Yao: Evaluation of the application of biological hemostatic dressings and gauze in surgical wounds. Chinese Tissue Engineering Research and Clinical Rehabilitation Vol.14(2010), p. 9636.

[9] Xiangfei Wang, Xiaodan Zhang and Hanxin Zhou: Biomedical research and clinical application of absorbable hemostatic materials. China Clinical Rehabilitative Tissue Engineering Research Vol.14(2010), p. 3974. 
[10] Liewen Lin: Tai Ling for pancreatectomy in prevention of pancreatic fistula. Medical Information Vol. 5(2011), p. 233.

[11] Lixia Huang: Naka Taiaya's application of laparoscopic resection of uterine fibroids. Medical Information Vol. 5(2011), p. 195

[12] Shiqiang Yang: Surgery for achilles tendon rupture tailing using . China Contemporary MedicIne Vol.18(2011), p. 49-52.

[13] HuachunLiu: Application of laminectomy tailing prevention of epidural scar adhesion. Chinese Contemporary Medicine Vol.18 (2011), p. 187-190.

[14] Hua Zhang:The application of Tai Ling on 150 cases of thyroid surgery. China Contemporary Medici-ne Vol.18(2011), p. 49-52. 